This is the final peer-reviewed accepted manuscript of:

Malaguti M, Cardenia V, Rodriguez-Estrada MT, Hrelia S, "Nutraceuticals and physical activity: Their role on oxysterols-mediated neurodegeneration"

which has been published in final form in

JOURNAL OF STEROID BIOCHEMISTRY AND MOLECULAR BIOLOGY 2019, vol. 193

(October), article n. 105430

ISSN 0960-0760

The final published version is available online at:

https://doi.org/10.1016/i.jsbmb.2019.105430

(C) 2019 Elsevier. This manuscript version is made available under the Creative Commons Attribution-NonCommercial-NoDerivs (CC BY-NC-ND) 4.0 International License

(http://creativecommons.org/licenses/by-nc-nd/4.0/) 


\title{
Nutraceuticals and physical activity: Their role on oxysterols-mediated neurodegeneration
}

\author{
Marco Malaguti $^{\text {a, } 1 \text {, *, Vladimiro Cardenia }}{ }^{\text {b, } 1}$, Maria Teresa Rodriguez-Estrada ${ }^{c}$, Silvana Hrelia ${ }^{\text {a }}$ \\ a Department for Life Quality Studies, Alma Mater Studiorum University of Bologna, Rimini, 47921, Italy \\ ${ }^{b}$ Department of Agricultural, Forest and Food Sciences DISAFA, University of Turin, Largo Braccini 2, 10095, Grugliasco, Italy \\ c Department of Agricultural and Food Sciences, Alma Mater Studiorum University of Bologna, Bologna, 40127, Italy
}

\section{ARTICLE INFO}

Keywords:

Oxysterols

Neurodegenerative diseases

Nutraceuticals

Physical activity

Bioactive compounds

\begin{abstract}
A B S T R A C T
Over the past few years, the contribution of oxysterols to the onset and development of some of the major neurodegenerative diseases (such as Alzheimer's and Parkinson's diseases) has been scientifically asserted, being mainly related to altered brain cholesterol homeostasis. To counteract oxysterol induced inflammation at neuronal level, one possible intervention approach is the administration of some nutrients and/or plant secondary metabolites. On the other hand, the pleiotropic beneficial effects of physical activity seem to play an important role on prevention and counteraction of neurodegenerative diseases, through the modulation of oxysterol homeostasis and the prevention of demyelination. The present review provides a picture of the promising role of nutraceuticals and physical activity on oxysterol-mediated neurodegeneration, pointing out also the different in vitro and in vivo aspects that need to be further investigated for a better understanding of the association of these three counterparts and their overall effect on people at increased risk for neurodegenerative diseases.
\end{abstract}

\section{Introduction}

To date, neurodegenerative diseases represent one of the greatest threats to public health, as well as one of the major economical burdens of healthcare systems. In 2015, the costs of dementia alone (to which Alzheimer's disease (AD) contributes with $60-70 \%$ of cases) accounted for $1.1 \%$ of the global gross domestic product [1] and it is expected that, in 2050, there will be a 3-fold increase of dementia cases [2]. The onset and progression of neurodegenerative diseases is mainly related to the altered brain cholesterol homeostasis and to systemic oxidative stress (OS). The latter can promote the oxidation of cholesterol and thus the formation of oxysterols, which can permeate the blood-brain barrier $(\mathrm{BBB})$ and play a pivotal role on oxidation-related neuropathologies of the brain [3-5]. Oxysterols generate an oxidative/inflammatory cascade, which increases their concentrations in the brain; such increase has been, in fact, associated with Alzheimer's (AD) [6] and Parkinson's diseases (PD) [7]. In particular, the balance between 24(S)-hydroxycholesterol (24-HC) and 27-hydroxycholesterol (27-HC) has been identified as a key aspect for brain cholesterol homeostasis. The accumulation of other oxysterols, such as 7-ketocholesterol (7-
$\mathrm{KC}$ ), appears to induce important mitochondrial dysfunctions (including alterations of oxidative phosphorylation, loss of mitochondrial membrane potential and the cytoplasmic release of the cytochrome c), which can lead to cell death [8-11]. In fact, there is plenty of evidence suggesting that mitochondrial dysfunction also plays a critical role in neurodegenerative diseases and neurodegeneration cascades; the organelles' malfunction could be mainly due to abnormal mitochondrial DNA (deletions and point mutations), mutated nuclear proteins that directly or indirectly interact with mitochondria, and OS $[12,13]$.

To counteract oxysterol induced inflammation at the neuronal level and prevent demyelination, besides the pharmacological treatments currently being used, any supporting approach should take into account OS modulation in order to limit the in vivo oxidative process; in this respect, some potential supporting strategies could be the administration of nutrients and/or plant bioactives that, together with continuous moderate exercise, could help in modulating mitochondrial OS. Moreover, exercise can further exert a neuro-protective action by triggering some neurobiological mechanisms (i.e. neurotransmitter secretion, hormones, neurotrophic factor levels) $[14,15]$, raising the concentration of several growth factors involved in brain tissue develop-

\footnotetext{
* Corresponding author.

Email address: marco.malaguti@unibo.it (M. Malaguti)

1 Both authors contributed equally to this work.
} 
Table 1

Oxysterol involvement in the main neurodegenerative diseases (AD, PD, MS, HD, and X-ALD) in diverse experimental systems (in vitro, in vivo and human studies).

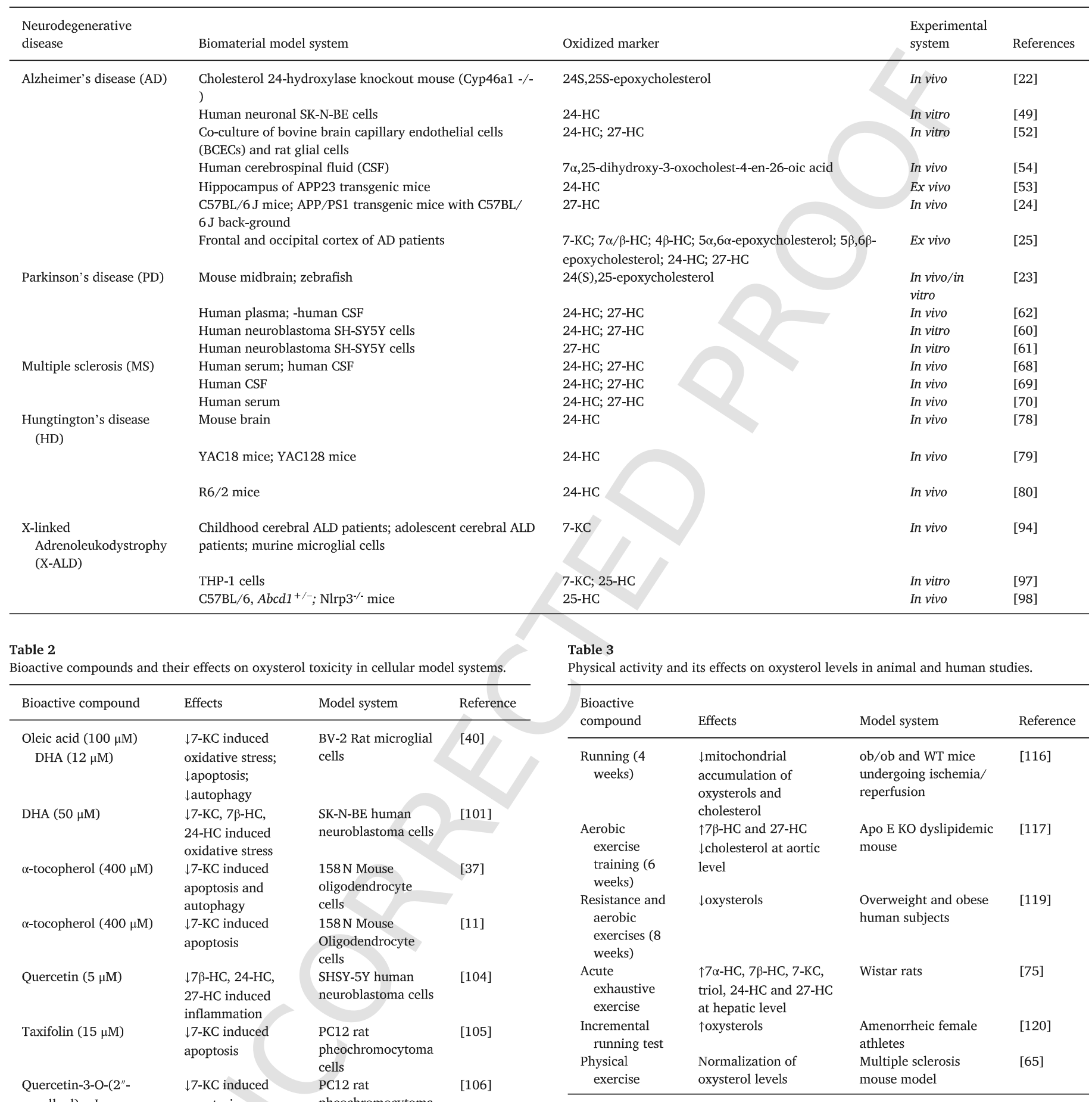

galloyl)- $\alpha$-L-

rhamnopyranoside apoptosis

pheochromocytoma

cells

$$
(25 \mu \mathrm{M})
$$

ment [16-18], modulating apoptosis and inducing neurogenesis in defined brain areas [19].

The present review depicts the promising role of nutraceuticals and physical activity on oxysterol-mediated neurodegeneration, aiming at laying the groundwork for a better understanding of the link between these three aspects and their overall impact on neurodegenerative diseases.

\section{Methods}

The Web of Science Core Collection (WOS) platform from Clarivate Analytics, Scopus from Elsevier and PubMed from MEDLINE were the databases selected. The following terms were used in the search strategy: Title and Abstract: ((oxysterol* OR oxidative stress OR bioactive compound* OR nutraceutical* OR physical activity) AND (brain OR brain disease* OR Alzheimer OR Parkinson OR Multiple sclerosis OR Dementia OR Hungtington OR Adrenoleukodystrophy)). The search was carried out using title and abstract fields only, in order to in- 
crease the accuracy, while the asterisk was used to include all the words derived from the same root (such as oxysterols from the same root "oxysterol"”). The search was mainly limited to the last five years, even though important and relevant earlier publications were also taken into consideration.

\subsection{Oxysterols and their role in brain diseases}

The brain cholesterol is synthesized de novo and its excess is immediately converted to oxidized forms (oxysterols), in particular 24-HC which is able to pass through the $\mathrm{BBB}$ reaching the peripheral circulation (2-6 mg/24h) [20,21]. 24-HC is mostly generated by cytochrome P450 (CYP46A1) activity, which is expressed in neurons, in healthy human brains and also in glial cells in Alzheimer's disease (AD) patients [22]. Oxysterols also act as ligands to nuclear receptors, while the side-chain hydroxy-, oxo-, epoxy- or carboxylate group of oxysterols can act as ligands to the liver $\mathrm{x}$ receptors (LXR) that are expressed in the brain, which induce the expression of ATP-binding cassette, sub-family A member 1 (modulator of cholesterol efflux); in addition, oxysterols can induce neurogenesis of dopaminergic neurons [23]. On the other hand, (25R)26-hydroxycholesterol (also known as 27-hydroxycholesterol (27-HC)) is the most circulating oxysterol in plasma (57.7-247.9 $\mathrm{ng} / \mathrm{mL}$ in healthy subjects) [24] and it flows from circulation into the brain passing through the BBB [25]. 27-HC is also present in the brain, where it acts as a sensitive modulator of cholesterol metabolism disorder by suppressing cholesterol synthesis and stimulating cholesterol transport in astrocytes [26]. It is well reported that disorders in brain cholesterol homeostasis could lead to neurodegenerative diseases.

Being recently and extensively reviewed [27], many pathologies are characterized by impaired levels of different oxysterols [28]. Among other oxysterols, 7-KC appears to be one of the most involved in numerous age-related pathological conditions. 7-KC accumulation has been correlated to the development of different types of cancers [29] and MS [30]; several studies confirm its implication in atherosclerosis [31], retinal diseases and Alzheimer's disease (AD) [25,32]. In cellular model systems derived from neuronal tissues, 7-KC appears to exert its effects through multiple mechanisms: i) disruption of redox homeostasis, ii) induction of inflammation, iii) modification of mitochondria, peroxisome and other organelles' functions, iv) induction of apoptosis and autophagy, and v) disruption of lipid metabolism [27]. It has been confirmed that 7-KC can induce an increase of ROS and nuclear factor kappa-light-chain-enhancer of activated $B$ cells $(\mathrm{Nf} \kappa \mathrm{B})$ in neuronal differentiated PC12 cells [33]. Nury et al. [34] and Zarrouk et al. [35] demonstrated that 7-KC treatment induced OS in cultured oligodendrocytes, by increasing intracellular levels of superoxide anion and hydrogen peroxide. Moreover, Leoni et al. [8] reported that 7-KC-induced OS is related to the loss of mitochondrial potential as evidenced by the decrease of ATP and NAD + production at mitochondrial respiratory chain level. In a study about the proinflammatory effect of 7-KC [36], the induction of cytokines (IL-18 and IL-1 $\beta$ ) in human primary microglial cells, was observed. 7-KC is also responsible for apoptosis induction not only in numerous cell culture models, but also in Caenorhabditis elegans. Finally, some evidence suggest that 7-KC triggers also autophagy [37,38]. Since the early 2000 's, some authors have described the particular cell death mechanism induced by $7-\mathrm{KC}$ in cell culture models (such as $158 \mathrm{~N}$ oligodendrocytes and BV- $2 \mathrm{mi}-$ croglial cells) as oxiapoptophagy, a term proposed to describe the concurrent induction of OS, apoptosis and autophagy leading to cell death $[34,37,39,40]$. On the other hand, other authors $[41,42]$ have reported that inflammation-induced oxysterols affected the in vitro and in vivo glia cell activation (BV2 microglial cell, primary microglia and astrocytes), even though the in vivo changes were less marked. In addition, since oxysterols are involved in glutamate and GABA receptors modulation, whose changes contribute to pathogenesis of $\mathrm{AD}$ and glau- coma, some authors have encouraged the use of oxysterols and neurosteroids as potential targets for these disorders [43].

Hence, neurons have a high demand for cholesterol to develop and maintain membrane-rich structures like axons, dendrites and synapses [44]; consequently, oxysterols play a key role in many different neurodegenerative diseases, which are divided in two main subfamilies: demyelinating and non-demyelinating neurodegenerative diseases $[25,32,45]$. Cholesterol is an essential component of myelin, thus its deficiency or lack of impacts the rate of myelin formation leading to motor symptoms [46]. Additionally, oxysterols inhibit myelin gene expression in the peripheral nervous system via LXR, $\alpha$ - and $\beta$-mediated signaling and also exert adverse effects on oligodendrocyte viability [47].

Table 1 summarizes the most recent studies on the involvement of oxysterols in the main neurodegenerative diseases (AD, Parkinson, etc).

\subsection{Alzheimer's disease $(A D)$}

$\mathrm{AD}$ is an irreversible neurodegenerative process which progressively destroys memory and other functions [48]. In $\mathrm{AD}$, it is widely reported that both 24-HC and 27-HC play a key role in the dysfunction of nerve cells and the alteration of BBB [49]. AD is characterized by a massive neurodegenerative process resulting mainly from the abnormal accumulation of different forms of $\beta$-amyloid peptides in various brain areas and around cerebral microvessels [50], due to the reduced ability of BBB to regulate the cerebral pool of these peptides. Björkhem et al. [21,51] hypothesized that production of $\beta$-amyloid in the brain is related to the balance between 24-HC and 27-HC; in fact, the level of 27-HC in the brains of $\mathrm{AD}$ patients was higher and its serum level was strongly correlated with that of cholesterol, thus suggesting a link between hypercholesterolemia and $\mathrm{AD}$. On the other hand, it is well known that LXR activation affects the transport of $\beta$-amyloid peptides through $\mathrm{BBB}$, since $\mathrm{ABCB} 1$, a transporter of $\beta$-peptides expressed at the luminal side of brain capillary endothelial cells (BCECs), is down-expressed in $\mathrm{AD}$ patients; however. its expression is increased by $24-\mathrm{HC}$ and 27-HC (LXR agonists) [52]. Recently, it has also been reported that oxysterols are involved in the progression of memory loss and cognitive impairment; in particular, a significant increase of $27-\mathrm{HC}$ in plasma of patients afflicted by mild cognitive impairment has been observed [24]. Again, the same authors have reported an increase and accumulation of $\beta$-amyloid (1-40) peptide (A $\beta 1-40)$ and $\beta$-amyloid (1-42) peptide (A $\beta 1-42)$ in both brain and plasma with consequent severe cognition effects related to enhanced 27-HC level. Djelti et al. [53] observed that the abundance of $\beta$-amyloid peptides with inhibition of CYP46A1, which encodes 24-hydroxylase, was more widespread in the APP23 mouse model of AD than in normal mice. Griffiths et al. [54] reported a significant reduction of $7 \alpha, 25$-dihydroxy-3-oxocholest-4-en-26-oic acid in CSF of AD patients. It is also well documented that 24-HC and 27-HC govern the expression of inflammatory cytokines and boost OS to induce neuronal apoptosis in $\mathrm{AD}[25,55]$.

\subsection{Parkinson's disease (PD)}

PD is characterized by a loss of dopaminergic neurons in the substantia nigra and intraneuronal $\alpha$-synuclein inclusion; however, since misdiagnosis is common, there is an urgent need of reliable biomarkers [45]. PD aetiology is mainly associated with mitochondrial dysfunction ascribable to OS and an increase in misfolded proteins, which have a central role in dopaminergic neurodegeneration [45,56,57]. Mitochondrial dysfunction is due to a deficiency in complex I activity of the respiratory chain, which leads to increased reactive oxygen species (ROS) generation as well as decreased ATP production; iron accumulation has also been reported in the substantia nigra of the brains of PD patients with consequent increased ROS [56]. Both non-enzymatic and enzy- 
matic oxidation of cholesterol lead to oxysterols production within tissues; cholesterol autoxidation mainly occurs in the sterol ring, giving rise to the formation of $7-\mathrm{HCs}(\alpha$ and $\beta$ ), 7-ketocholesterol (7-KC), 5,6 -epoxides ( $5 \alpha, 6 \alpha$-EC and $5 \beta, 6 \beta$-EC), and triol [58]. On the other hand, it has been reported that the use of drugs affecting cholesterol metabolism (such as statins), reduce $\alpha$-synuclein aggregation, thus confirming the involvement of cholesterol homeostasis in PD [11]. It has also been reported that an accumulation of $\alpha$-synuclein protein is linked to concurrent reduction of tyrosine hydroxylase, which has a key role in dopamine synthesis [59]. Several studies $[60,61]$ have documented that $27-\mathrm{HC}$ reduces the expression of tyrosine hydroxylase and increases the level of $\alpha$-synuclein protein in human neuroblastoma cells, while $24-\mathrm{HC}$ increases the level of tyrosine hydroxylase. It should be pointed out that different results have been reported about circulating 24-HC level of PD patients. Thus, main attention has to be paid to the level of 24-HC and 27-HC in both plasma and cerebrospinal fluid (CSF). In fact, Bjorkem et al. [62] found a higher 24-HC level in CSF of PD patients than in controls and the levels were highly correlated to disease duration; on the other hand, no correlation to the disease was observed for 27-HC, even though it displayed increased levels in CSF. However, these results confirm a defect of BBB function.

Recently, clinical management of PD has moved toward the use of antioxidants or bioactive compounds able to counteract OS. In fact, animal models have shown promising results in the use of antioxidants and flavonoids on mitochondrial dysfunction. Seidl et al. [63] demonstrated that the onset of PD can be delayed by the consumption of dietary supplements rich in vitamin $E$. The combination of coenzyme $Q_{10}$ with vitamin $\mathrm{E}$ or creatine has also been successfully tested, showing a significant reduction in the depletion of dopamine, loss of tyrosine hydroxylase neurons, lipid peroxidation and $\alpha$-synuclein aggregation in the neurons [64]. On the other hand, the intake of vitamin $C$ and flavonoids did not reveal any significant beneficial effect in either the prevention or the treatment of PD [64]. Thus, more antioxidant approaches need to be tested in order to better clarify if OS is a downstream effect of mitochondrial dysfunction rather than a direct cause of PD-related neurodegeneration [56].

\subsection{Multiple sclerosis}

Multiple sclerosis (MS) is a chronic, inflammatory and degenerative disease characterized by a central nervous demyelinating process with cyclic loss and repair of myelin sheaths associated with chronic neuronal loss $[64,65]$. The latter is accompanied by modified levels of oxysterols in the brain, blood and cerebrospinal fluid of MS patients, while the accumulation of such derivatives is believed to participate in the onset and progression of the disease through their implication in inflammation, OS, demyelination and neurodegeneration [65].

It might be pointed out that the increase of blood cholesterol level induces an increase of inflammation within the central nervous system (CNS) through the disruption of BBB and subsequent entry of 27-HC, thus increasing both immune recruitment and oxidative damages [66]. In fact, in MS patients, altered levels of oxysterols have been demonstrated to be strictly correlated with demyelinating episodes; a reduced 25-hydroxycholesterol (25-HC) synthesis, by depletion of 25-hydroxylase activity, significantly attenuates disease outcomes by limiting the entry of T lymphocytes in CNS [67]. Again, decreased levels of both 24-HC and 27-HC in CSF have been found in MS patients [68]. Similar results were also found in CSF and serum by Vuletic et al. [69] and Teunissen et al. [70], respectively. Moreover, serum has also showed a higher level of 24-HC during the active neurodegeneration of demyelination, while a lower level has appeared after losing a quantitatively higher amount of cell mass [70].

In addition, 7-KC induces neuronal damage with activation and migration of microglial cells [71], while $7 \beta-\mathrm{HC}, 7-\mathrm{KC}$ and $24-\mathrm{HC}$ could trigger oligodendroglial cell death [72]. In that context, cholesterol homeostasis could be a promising strategy and, as stated for other neurodegenerative processes, the use of statins could exert some benefit. Statins could have potential therapeutic implication not only for strokes but also in neurological disorders (such as $\mathrm{AD}, \mathrm{PD}$, MS and primary brain tumors); they have been used for lowering cholesterol but, besides their anti-atherosclerotic and cardio-protective effects, compelling clinical and preclinical studies delineate the neuro-protective efficacy in all of these neurological disorders [73]. To date, the neuroprotective mechanism of statins is not completely clear; however, the reduction of ROS generation, the impairment of $\beta$-amyloid production and serum apolipoprotein $\mathrm{E}$ levels, the enhancement of endothelial nitric oxide synthase levels and cerebral blood flow, and modulation of cognitive related receptors and matrix metallo proteases, seem to be involved in the neuroprotection.

As described before, OS plays a key role in the pathogenesis of several neurodegenerative diseases. ROS and reactive nitrogen species (RNS) generated by monocytes, macrophages and activated microglia act as mediators of neurodegeneration and axonal damage typical of these disorders [64]. Considering the implications of mitochondrial dysfunction and OS in the onset and progression of these neurodegenerative diseases, useful innovative strategies should be developed based on organelle dynamics, bioenergetics, ROS scavenging and OS counteraction $[26,64,74,75]$.

\subsection{Hungtington's disease (HD)}

The Huntington's disease (HD) is an autosomal dominant neurodegenerative disorder, which is caused by expanded CAG trinucleotide repeat (TNR) in N-terminus of the huntingtin protein (HTT) [76,77]. Thus, huntingtin is produced with abnormal long polyglutamine sequences that lead to protein fragmentation and consequent neuronal dysfunction [76].

Typical symptoms consist of dystonia, cognitive decline, motor disorder such as involuntary movement and impairment of voluntary movements (e.g. incoordination and bradykinesia) [59]. However, in addition to cognitive and motor disorder, major depression is also common in 50\% of HD patients, as well as irritability and apathy [76]. There is considerable evidence indicating that, when HD occurs, the cholesterol metabolism is strongly affected [78-80]. In HD rodent models, a significant decrease of cholesterol precursors (lanosterol, lathosterol and desmosterol) levels was found in the brain before the onset of motor defects, while cholesterol decreased later on $[78,79]$. Again, the same authors found a significant correlation between synthesis rates of cholesterol and 24-HC in the brain, which confirms the consistent cholesterol biosynthesis defects and points to $24-\mathrm{HC}$ in plasma as a biomarker of brain cholesterol metabolism [78]. On the other hand, a significant correlation between lipid peroxidation in plasma and degree of severity in HD patients was also found, suggesting plasma malondialdehyde (MDA) as a biomarker for evaluating treatment efficacy [81]. As reported [82], cholesterol supplementation delivered to the brain through polymeric nanoparticles (polylactide-co-glycolide; PLGA) able to cross BBB, could be a useful strategy for HD treatment. In addition, an increase of CYP46A1 (enzyme involved in the $24-\mathrm{HC}$ synthesis) in neurons could stimulate cholesterol metabolism.

It might be pointed out that neurons exhibit a high level of sensitivity and susceptibility to oxidative damage, probably due to the low presence of glutathione (GSH) and antioxidants enzymes [83]. On the other hand, HTT is universally expressed in the body, but cell degeneration occurs only in the striatum and cortex; the mechanisms for the selection of neuronal death are multifaceted and different studies suggest that OS and mitochondria dysfunction play a key role in the oxidative damage of proteins, lipids and DNA $[81,84,85]$. Different stud- 
ies have revealed a correlation between HD and the increase of MDA and 8-hydroxy-2'-deoxyguanosine (8-OHdG) in plasma [81], as well as elevated levels of lipid peroxides and lactate [85]; again, heme oxygenase, 3-nitrotyrosine and MDA were elevated in the striatum, cortex and serum of HD patients [86-88]. Thus, the use of antioxidants and essential fatty acids could be a potential therapeutic strategy for the treatment of HD in order to counteract ROS production, which affect neuron survival [83]. In R6/2 mice, the supplementation with creatine, vitamin C, coenzyme Q, tauroursodeoxycholic acid (TUDCA), docosahexenoic acid (DHA) and eicosapentenoic acid (EPA) increased the life span and motor performance in association with reduced polyQ aggregated in the brain [84]. When rats are treated with 3-nitropropionic acid (3-NP), which is a neurotoxin that reproduces the neurodegenerative effects of $\mathrm{HD}$, their motor and cognitive impairments are reduced if they receive melatonin, curcumin or carvedilol; lycopene has also proved to be able to reduce OS markers and to improve behavior in 3-NP rat model of HD [89-92]. Recent literature suggests neurogenesis as important tool for regenerative therapy of the HD brain, since neurogenesis could be upregulated by numerous antioxidants. Therefore, the use of antioxidants might provide neurotropic, proneurogenic and neuroprotective support for HD brain [93].

\subsection{X-linked adrenoleukodystrophy (X-ALD)}

X-linked adrenoleukodystrophy (X-ALD), which includes cerebral demyelinating and inflammatory childhood phenotypes, is a genetic disorder induced by a mutation in the ABCD1 gene with consequent accumulation of very long-chain fatty acids (VLCFA; > C22) in tissues including brain white matter, the spinal cord, adrenal cortex and plasma [94]. Since OS also characterizes X-ALD, GSH, antioxidants (such as vitamin E), DHA and oxysterols have been proposed as markers for determining disease progression stage [95]. In particular, main attention has been addressed to 7-KC. Considering that C7 oxysterols (in particular 7-KC) increase in tissue and plasma of X-ALD patients [96] and that 7-KC induces inflammation, OS and cell death [97], some researchers have investigated the impact of $7-\mathrm{KC}$ on the peroxisomal status of microglia cells. Nury et al. [94] confirmed that 7-KC is able to induce cell death in microglia cells and generalized peroxisomal modifications inducing peroxisomal dysfunction and brain damage, which contribute to X-ALD development. Again, Jang et al. [98] in childhood cerebral ALD identified an aberrant production of cholesterol 25-hydroxylase and 25-HC, which promoted robust NLRP3 inflammasome assembly, mitochondrial ROS and LXR-mediated pathways. The latter highlights that 25-HC mediates the neuroinflammation of X-ALD through NLRP3 inflammasome activation. Considering that OS plays a key role in the progression of X-ALD, the use of antioxidants as strategy to counteract this disease has been investigated in depth. Some authors [99] have promoted the use of valproic acid as promising therapeutic approach for $\mathrm{X}$-ALD. In fact, the valproic acid (anti-epileptic drug) is able to induce the expression of $\mathrm{ABCD} 2$ peroxisomal transporter, reducing the oxidative damage. Others [100] have proved the ability of $\mathrm{N}$-acetyl-cisteine, $\alpha$-lipoic acid and $\alpha$-tocopherol to scavenge VLCFA-dependent ROS generation, establishing the link between OS and axonal damage. Early and carefully tailored antioxidant intervention could be a therapeutic option to reduce the effect of the disease.

\subsection{Prevention of oxysterols toxicity at neuronal and glial level by natural bioactive compounds}

Since the relationship between neurodegenerative diseases (such as $\mathrm{AD}$ ) and oxysterols has been asserted, different studies, mainly conducted in neuronal and glial model systems, have demonstrated that some nutrients and plant bioactives are able to counteract oxysterol and in particular 7-KC effects [27].
Debbabi et al. [40] evaluated the possibility to counteract 7-KC induced oxyapoptophagy in BV-2 murine microglial cells. The induction of OS, inflammation and apoptosis at the microglial level is now considered a major issue leading to neurodegeneration. Debbabi and collaborators demonstrated that specific fatty acids such as oleic acid (OA) and docosahexaenoic acid (DHA), two fatty acids typically present in the Mediterranean diet, can prevent 7-KC induced cell damage in BV-2 cultures by counteracting OS [40]. In fact, the data demonstrated that both OA and DHA reduced the percentage of dihydroethidium positive cells (a biomarker of superoxide anion production) and that OA was also able to prevent 4-hydroxynonenal overproduction after 7-KC treatment. The study by Debbabi et al. [40] also showed the protective effect of these two fatty acids against apoptosis, autophagy and membrane fluidity impairment. In a different study on SK-N-BE cell line (human neuroblastoma), a protective effect of $50 \mu \mathrm{M}$ of DHA against OS induced by different oxysterols (such as $7-\mathrm{KC}, 7 \beta-\mathrm{HC}$, and $24-\mathrm{HC}$ ), was also confirmed [101]. In oligodendrocyte cultures, $20 \mu \mathrm{g} / \mathrm{mL}$ of $7-\mathrm{KC}$ induced apoptosis by activating caspase- 3 and inducing DNA fragmentation, associated with loss of transmembrane mitochondrial potential, dephosphorylation of Akt protein and glycogen synthase kinase 3 beta (GSK3 $\beta$ ), and the degradation of the induced myeloid leukemia cell differentiation protein (Mcl-1) [11]. The authors demonstrated that co-treatment with $400 \mu \mathrm{M}$ of $\alpha$-tocopherol was able to counteract 7-KC induced apoptosis by inhibiting Akt and GSK3 $\beta$ dephosphorylation and Mcl-1 degradation. Likewise, in a different study that also used oligodendrocyte as model system, the $\alpha$-tocopherol protective effect against 7-KC treatment was associated with a reduced incorporation of this oxysterol in lipid rafts [102]. In $158 \mathrm{~N}$ murine cells, $400 \mu \mathrm{M}$ of $\alpha$-tocopherol were able to counteract 7-KC induced apoptosis as shown by the reduction of specific biomarkers, such as mitochondrial depolarization and nuclei fragmentation [37]. In the same study, the authors clearly demonstrated that vita$\min \mathrm{E}$ is able to prevent the oxiapoptophagy process induced by 7-KC by showing, through Western blot analysis, that vitamin E reduced the conversion of LC3-I into LC3-II, a commonly used biomarker of autophagy. The $\alpha$-tocopherol protective effect has been recently confirmed by the same research group in a study where they demonstrated that 7-KC increases autophagy biomarkers and induces peroxisomal changes also at concentrations that do not induce apoptosis [103].

Besides specific fatty acids and vitamin E, some studies have investigated the potential protective effect of plant secondary metabolites against oxysterol toxicity at the neuronal level. When some researchers treated neuroblastoma SHSY-5Y cells with a mixture of $7 \beta-\mathrm{HC}, 24-\mathrm{HC}$ and 27-HC (at a $5 \mu \mathrm{M}$ concentration each), or with the three oxysterols alone, they observed an increased expression of inflammatory mediators (such as CD36, B1-integrin, IL-8, MCP-1, MMP-9), via the induction of TLR4 and COX2 [104]. However, 1-h pretreatment with $5 \mu \mathrm{M}$ quercetin, a flavonol abundant in many fruits and vegetables, loaded into nanoparticles, was able to counteract the increase of these biomarkers, as well as the expression of COX2 and TLR4 [104]. Taken together, this data suggests the possibility to counteract oxysterols induced inflammation at the neuronal level through quercetin administration. In another study, Kim et al. [105] demonstrated that taxifolin, a flavanonol present in some plant trees such as Cedrus deodara, Pinus roxburghii and some varieties of Taxus chinensis, is able to positively affect apoptotic biomarkers and prevent cell death in 7-KC treated PC12 cells. Similarly, a quercetin derivate, quercetin-3-O-(2"-galloyl)- $\alpha$-L-rhamnopyranoside, prevented 7-KC induced apoptosis in differentiated PC12 cells [106]; in this case, it was suggested that the quercetin derivate might suppress the activation of both the intrinsic apoptotic and the caspase-8-dependent pathways, and that the protective effect could be related to oS counteraction. Table 2 summarizes the studies describing the effects of each bioactive compound and the model system used. 
In another study [9], the administration of Raphanus sativus $c v$ Sango sprout juice (SSJ) (75 mg/kg b.w. SSJ/day), rich in sulforaphane, raphasatin and anthocyanins, was able to counteract brain oxysterol formation in rats (non-genetic model) subjected to a high-fat (34\% crude fat) dietary regimen.

The research path for the identification of new bioactive natural molecules able to counteract oxysterol toxicity in neuronal and glial cells has been opened and more studies are necessary to recognize whether natural compounds may hold benefits in preventing and controlling the oxidative/inflammatory cascade generated by oxysterols in vitro and in vivo.

\subsection{Physical activity counteracts oxysterols toxicity}

Regular physical activity is now recognized as an essential component of a healthy life style, due to its pleiotropic beneficial effects on different human tissues (such as muscles, heart, vascular and brain), leading to prevention and counteraction of many different chronic pathological conditions, including cardiovascular, metabolic and neurodegenerative diseases [107]. Exercise has been demonstrated to improve spatial learning, working memory, cognitive and executive function $[108,109]$. Moreover, it induces both acute and chronic biological effects on many brain areas by triggering numerous neurobiological mechanisms, such as modulating neurotransmitters secretion [15], modulating hormones and neurotrophic factor levels [14]. Exercise increases the levels of numerous growth factors involved in correct brain tissue development, such as fibroblast growth factor-2 (FGF-2) [110], insulin-like growth factor-1 (IGF-1) [16], vascular endothelial growth factor (VEGF) [17], and brain derived neurotrophic factor (BDNF) [18]. The modulation of these factors, together with the reduction of OS at brain level [111], the modulation of apoptosis and the induction of neurogenesis in specific areas of the brain [19], seems to be responsible for the protective effects of exercise toward neurodegenerative diseases [14]. The effects of physical exercise and training on cholesterol and oxysterols homeostasis and thus the counteraction of oxysterol toxicity, are still not completely understood and thus need more study; however, physical activity seems to play a crucial role in the counteraction of numerous pathological conditions and chronic/degenerative diseases, in particular cardiovascular diseases.

Cholesterol and oxysterols, such as $7 \alpha-\mathrm{HC}, 7 \beta-\mathrm{HC}$, 7-KC, accumulate at cardiac level during ischemia/reperfusion events [112-114]. Oxysterol build-up leads to lipid peroxidation and mitochondrial dysfunction; in fact, it has been proposed that reduction of mitochondrial cholesterol during reperfusion might protect mitochondria [115]. A recent study performed with both wild-type and ob-ob mouse models, demonstrated that regular physical exercise (running on a treadmill $0.5-1 \mathrm{~h} /$ day for 4 weeks) was able to improve heart antioxidant capacity, reduce mitochondrial cholesterol accumulation and its subsequent oxidation to oxysterols (such as $7 \alpha-\mathrm{HC}, 7-\mathrm{KC}, 5 \alpha, 6 \alpha-\mathrm{EC}$ ), and, in the final analysis, preserve mitochondrial function after ischemia/reperfusion [116].

In another study carried out with an Apo E KO dyslipidemic mouse model, it was observed that aerobic exercise training increases $7 \beta-\mathrm{HC}$ and 27-HC levels in the aortic arch by increasing Cyp27a1, Cd36 and reducing Cyp7b1 expression, thus contributing to atherogenesis prevention [117]. In fact, Cd36 has been demonstrated to be involved in lipid clearance associated with reverse cholesterol transport [118].

Moreover, it has been demonstrated that a regular combination of aerobic and resistance exercises reduce both $7 \alpha-\mathrm{HC}$ and $7 \beta-\mathrm{HC}$ serum levels and improve cholesterol homeostasis in obese human adults [119].

If, on one hand, exercise training seems to represent an intervention able to counteract cholesterol oxidation in both animals and hu- mans, on the other hand acute exhaustive exercise does not exert similar beneficial effect. We actually demonstrated that exhaustive exercise session is a prooxidant stimuli that induces cholesterol oxidation in rat liver; in fact, $7 \alpha-\mathrm{HC}, 7 \beta-\mathrm{HC}, 7-\mathrm{KC}$, triol, $24-\mathrm{HC}$ and $27-\mathrm{HC}$ increased in rat liver after a single treadmill exercise bout till exhaustion [75]. Ayers et al. [120] had previously proposed the idea that strenuous exercise could induce cholesterol oxidation, as they found that strenuous treadmill running increases plasma oxysterols level in amenorreic female.

Although evidence supporting the possibility to counteract negative oxysterol effects on cardiac and vascular tissues is increasing, only few authors have proposed a relationship between physical activity and oxysterols at brain tissue level. A recent review [65] proposed a role for physical activity in the modulation of oxysterol homeostasis at the brain level in MS mouse models; in fact, they consider that abnormal oxysterol accumulation contributes to the onset and progression of the disease, due to oxysterol implication in OS, inflammation, and neurodegeneration. Moreover, the authors have suggested that physical activity may counteract oxysterol toxicity thanks to its beneficial effects on neuronal plasticity, inflammation and OS, which also leads to an improvement of blood-brain integrity with an overall positive effect for brain health [65]. Other authors have established that moderate continuous exercise could be potentially useful to enhance myelin sheath regeneration and improve the concentration of myelination-related growth factors and cytokines; its practice is, thus, encouraged to both prevent and treat demyelination [121]. Table 3 summarizes the studies describing the effects of physical exercise and the model system used.

\section{Concluding remarks}

As emphasized along this review, oxysterols have proved to play a key role on the onset and development of many neurodegenerative diseases, which is one of the greatest threats to public health of the 21 st century. Since mitochondrial dysfunction, altered brain cholesterol homeostasis and systemic OS are involved in the progression of neurodegenerative diseases, potential supporting strategies to traditional pharmacological treatments (such as dietary administration of nutraceuticals and moderate physical activity) should focus on modulation of OS. In particular, nutraceutical-containing nanosystems (i.e. liposomes, polymeric and inorganic nanoparticles) for targeted mitochondrial bio-delivery have a great potential as a supporting strategy, as they could overcome multiple barriers for targeting mitochondria, thus enhancing the bioactives' effects [122,123]. Tailored interventions with targeted nutraceuticals to reduce OS and to induce enzymes with an antioxidant/detoxifying activity, together with the triggering action of exercise on several growth factors involved in neurogenesis (i.e. FGF-2, IGF-1, VEGF, BDNF), can thus contribute to the improvement of the pathological profile of diverse oxidation-related brain neuropathologies.

However, despite being promising, the association between oxysterol homeostasis, physical activity and neurodegenerative diseases has still to be fully elucidated. Further research should be performed at both in vitro and in vivo levels, including randomized clinical trials and mitochondrial-targeted nutraceuticals, to be able to better understand the link between physical exercise, oxysterol levels, mitochondria dynamics, and clinical outcomes in people at increased risk for neurodegenerative diseases.

\section{Funding}

This work was supported by MIUR-PRIN 2015 (No. 20152HKF3Z) and by the Basic Research Funding (RFO 2018; Alma Mater Studiorum-Università di Bologna, Italy). 


\section{References}

[1] World Health Organization, Dementia, 2017 https://www.who.int/news-room/ fact-sheets/detail/dementia.

[2] Alzheimer's Disease International (ADI), Policy Brief for Heads of Government: The Global Impact of Dementia 2013-2050, London (UK), 2013.

[3] D. Lutjohann, O. Breuer, G. Ahlborg, I. Nennesmo, A. Siden, U. Diczfalusy, Bjorkhem, Cholesterol homeostasis in human brain: evidence for an age-dependent flux of 24S-hydroxycholesterol from the brain into the circulation, Proc. Natl. Acad. Sci. U. S. A. 93 (1996) 9799-9804.

[4] N. Aytan, T. Jung, F. Tamturk, T. Grune, N. Kartal-Ozer, Oxidative stress related changes in the brain of hypercholesterolemic rabbits, Biofactors 33 (2008) 225-236.

[5] G. Poli, F. Biasi, G. Leonarduzzi, Oxysterols in the pathogenesis of major chronic diseases, Redox Biol. 1 (2013) 125-130.

[6] M. Shafaati, A. Marutle, H. Pettersson, A. Lovgren-Sandblom, M. Olin, I. Pikuleva, B. Winblad, A. Nordberg, I. Bjorkhem, Marked accumulation of 27-hydroxycholesterol in the brains of Alzheimer's patients with the Swedish APP 670/671 mutation, J. Lipid Res. 52 (2011) 1004-1010.

[7] D. Cheng, A.M. Jenner, G. Shui, W.F. Cheong, T.W. Mitchell, J.R. Nealon, W.S Kim, H. McCann, M.R. Wenk, G.M. Halliday, B. Garner, Lipid pathway alterations in Parkinson's disease primary visual cortex, PLoS One 6 (2011), e17299.

[8] V. Leoni, T. Nury, A. Vejux, A. Zarrouk, C. Caccia, M. Debbabi, A. Fromont, R. Sghaier, T. Moreau, G. Lizard, Mitochondrial dysfunctions in 7-ketocholesterol-treated $158 \mathrm{~N}$ oligodendrocytes without or with alpha-tocopherol: impacts on the cellular profil of tricarboxylic cycle-associated organic acids, long chain saturated and unsaturated fatty acids, oxysterols, cholesterol and cholesterol precursors, J. Steroid Biochem. Mol. Biol. 169 (2017) 96-110.

[9] C. Prunet, S. Lemaire-Ewing, F. Menetrier, D. Neel, G. Lizard, Activation of caspase-3-dependent and -independent pathways during 7-ketocholesterol- and 7beta-hydroxycholesterol-induced cell death: a morphological and biochemical study, J. Biochem. Mol. Toxicol. 19 (2005) 311-326.

[10] A. Vejux, G. Lizard, Cytotoxic effects of oxysterols associated with human dis eases: induction of cell death (apoptosis and/or oncosis), oxidative and inflammatory activities, and phospholipidosis, Mol. Aspects Med. 30 (2009) 153-170.

[11] K. Ragot, D. Delmas, A. Athias, T. Nury, M. Baarine, G. Lizard, Alpha-Tocopherol impairs 7-ketocholesterol-induced caspase-3-dependent apoptosis involving GSK-3 activation and Mcl-1 degradation on $158 \mathrm{~N}$ murine oligodendrocytes, Chem. Phys. Lipids 164 (2011) 469-478.

[12] E. Lezi, R.H. Swerdlow, Mitochondria in neurodegeneration, Adv. Exp. Med. Biol. 942 (2012) 269-286

[13] M. Rango, N. Bresolin, Brain mitochondria, aging, and parkinson's disease, Genes (Basel) 9 (2018).

[14] G.F. Hamilton, J.S. Rhodes, Exercise regulation of cognitive function and neuroplasticity in the healthy and diseased brain, Prog. Mol. Biol. Transl. Sci. 135 (2015) 381-406.

[15] R. Meeusen, K. De Meirleir, Exercise and brain neurotransmission, Sports Med. 20 (1995) 160-188.

[16] Q. Ding, S. Vaynman, M. Akhavan, Z. Ying, F. Gomez-Pinilla, Insulin-like growth factor I interfaces with brain-derived neurotrophic factor-mediated synaptic plasticity to modulate aspects of exercise-induced cognitive function, Neuroscience 140 (2006) 823-833.

[17] N. Uysal, M. Kiray, A.R. Sisman, U.M. Camsari, C. Gencoglu, B. Baykara, C. Cetinkaya, I. Aksu, Effects of voluntary and involuntary exercise on cognitive functions, and VEGF and BDNF levels in adolescent rats, Biotech. Histochem. 90 (2015) 55-68.

[18] S. Vaynman, F. Gomez-Pinilla, License to run: exercise impacts functional plasticity in the intact and injured central nervous system by using neurotrophins, Neurorehabil. Neural Repair 19 (2005) 283-295.

[19] D.H. Kim, I.G. Ko, B.K. Kim, T.W. Kim, S.E. Kim, M.S. Shin, C.J. Kim, H. Kim, K.M. Kim, S.S. Baek, Treadmill exercise inhibits traumatic brain injury-induced hippocampal apoptosis, Physiol. Behav. 101 (2010) 660-665.

[20] L. Iuliano, P.J. Crick, C. Zerbinati, L. Tritapepe, J. Abdel-Khalik, M. Poirot, Y. Wang, W.J. Griffiths, Cholesterol metabolites exported from human brain, Steroids 99 (2015) 189-193.

[21] I. Bjorkhem, V. Leoni, P. Svenningsson, On the fluxes of side-chain oxidized oxysterols across blood-brain and blood-CSF barriers and origin of these steroids in CSF (Review), J. Steroid Biochem. Mol. Biol. (2018).

[22] A. Meljon, Y. Wang, W.J. Griffiths, Oxysterols in the brain of the cholesterol 24-hydroxylase knockout mouse, Biochem. Biophys. Res. Commun. 446 (2014) 768-774.

[23] S. Theofilopoulos, Y. Wang, S.S. Kitambi, P. Sacchetti, K.M. Sousa, K. Bodin, J. Kirk, C. Salto, M. Gustafsson, E.M. Toledo, K. Karu, J.A. Gustafsson, K.R. Steffensen, P. Ernfors, J. Sjovall, W.J. Griffiths, E. Arenas, Brain endogenous liver X receptor ligands selectively promote midbrain neurogenesis, Nat. Chem. Biol. 9 (2013) 126-133.

[24] X. Zhang, Y. Xi, H. Yu, Y. An, Y. Wang, L. Tao, Y. Wang, W. Liu, T. Wang, R. Xiao, 27-hydroxycholesterol promotes Abeta accumulation via altering Abeta metabolism in mild cognitive impairment patients and APP/PS1 mice, Brain Pathol. (2018).

[25] G. Testa, E. Staurenghi, C. Zerbinati, S. Gargiulo, L. Iuliano, G. Giaccone, F. Fanto, G. Poli, G. Leonarduzzi, P. Gamba, Changes in brain oxysterols at differ- ent stages of Alzheimer's disease: their involvement in neuroinflammation, Redox Biol. 10 (2016) 24-33.

[26] V. Cardenia, F. Vivarelli, S. Cirillo, M. Paolini, M.T. Rodriguez-Estrada, D. Canistro, Dietary effects of Raphanus sativus cv Sango on lipid and oxysterols accumulation in rat brain: a lipidomic study on a non-genetic obesity model, Chem. Phys. Lipids 207 (2017) 206-213.

[27] F. Brahmi, A. Vejux, R. Sghaier, A. Zarrouk, T. Nury, W. Meddeb, L. Rezig, A Namsi, K. Sassi, A. Yammine, I. Badreddine, D. Vervandier-Fasseur, K. Madani, L. Boulekbache-Makhlouf, B. Nasser, G. Lizard, Prevention of 7-ketocholesterol-induced side effects by natural compounds, Crit. Rev. Food Sci. Nutr. (2018) 1-20.

[28] W.J. Griffiths, J. Abdel-Khalik, E. Yutuc, A.H. Morgan, I. Gilmore, T. Hearn, Y. Wang, Cholesterolomics: an update, Anal. Biochem. 524 (2017) 56-67.

[29] G. Poli, F. Biasi, Potential modulation of cancer progression by oxysterols, Mol. Aspects Med. 49 (2016) 47-48.

[30] S. Mukhopadhyay, K. Fellows, R.W. Browne, P. Khare, S. Krishnan Radhakrishnan, J. Hagemeier, B. Weinstock-Guttman, R. Zivadinov, M. Ramanathan, Interdependence of oxysterols with cholesterol profiles in multiple sclerosis, Mult. Scler. 23 (2017) 792-801.

[31] M.A. Lyons, S. Samman, L. Gatto, A.J. Brown, Rapid hepatic metabolism of 7-ketocholesterol in vivo: implications for dietary oxysterols, J. Lipid Res. 40 (1999) 1846-1857.

[32] A. Zarrouk, A. Vejux, J. Mackrill, Y. O’Callaghan, M. Hammami, N. O’Brien, G. Lizard, Involvement of oxysterols in age-related diseases and ageing processes, Ageing Res. Rev. 18 (2014) 148-162.

[33] E.R. Jang, C.S. Lee, 7-ketocholesterol induces apoptosis in differentiated PC12 cells via reactive oxygen species-dependent activation of NF-kappaB and Akt pathways, Neurochem. Int. 58 (2011) 52-59.

[34] T. Nury, A. Zarrouk, J.J. Mackrill, M. Samadi, P. Durand, J.M. Riedinger, M. Do ria, A. Vejux, E. Limagne, D. Delmas, M. Prost, T. Moreau, M. Hammami, R. Delage-Mourroux, N.M. O'Brien, G. Lizard, Induction of oxiapoptophagy on 158N murine oligodendrocytes treated by 7-ketocholesterol-, 7beta-hydroxycholesterol-, or 24(S)-hydroxycholesterol: Protective effects of alpha-tocopherol and docosahexaenoic acid (DHA; C22:6 n-3), Steroids 99 (2015) 194-203.

[35] A. Zarrouk, T. Nury, E.M. Karym, A. Vejux, R. Sghaier, C. Gondcaille, P. Andreoletti, D. Trompier, S. Savary, M. Cherkaoui-Malki, M. Debbabi, A. Fromont, J.M. Riedinger, T. Moreau, G. Lizard, Attenuation of 7-ketocholesterol-induced overproduction of reactive oxygen species, apoptosis, and autophagy by dimethyl fumarate on 158N murine oligodendrocytes, J. Steroid Biochem. Mol. Biol. 169 (2017) 29-38.

[36] G. Shi, S. Chen, W.S. Wandu, O. Ogbeifun, L.F. Nugent, A. Maminishkis, S.J. Hinshaw, I.R. Rodriguez, I. Gery, Inflammasomes induced by 7-Ketocholesterol and other stimuli in RPE and in bone marrow-derived cells differ markedly in their production of IL-1beta and IL-18, Invest. Ophthalmol. Vis. Sci. 56 (2015) $1658-1664$.

[37] T. Nury, A. Zarrouk, A. Vejux, M. Doria, J.M. Riedinger, R. Delage-Mourroux, G. Lizard, Induction of oxiapoptophagy, a mixed mode of cell death associated with oxidative stress, apoptosis and autophagy, on 7-ketocholesterol-treated 158N murine oligodendrocytes: impairment by alpha-tocopherol, Biochem. Biophys. Res. Commun. 446 (2014) 714-719.

[38] A. Badreddine, A. Zarrouk, E.M. Karym, M. Debbabi, T. Nury, W. Meddeb, R. Sghaier, M. Bezine, A. Vejux, L. Martine, S. Gregoire, L. Bretillon, E. Prost-Camus, P. Durand, M. Prost, T. Moreau, M. Cherkaoui-Malki, B. Nasser, G. Lizard, Argan oil-mediated attenuation of organelle dysfunction, oxidative stress and cell death induced by 7-Ketocholesterol in murine oligodendrocytes $158 \mathrm{~N}$, Int. J. Mol. Sci. 18 (2017).

[39] S. Monier, M. Samadi, C. Prunet, M. Denance, A. Laubriet, A. Athias, A. Berthier, E. Steinmetz, G. Jurgens, A. Negre-Salvayre, G. Bessede, S. Lemaire-Ewing, D. Neel, P. Gambert, G. Lizard, Impairment of the cytotoxic and oxidative activitie of 7 beta-hydroxycholesterol and 7-ketocholesterol by esterification with oleate, Biochem. Biophys. Res. Commun. 303 (2003) 814-824.

[40] M. Debbabi, A. Zarrouk, M. Bezine, W. Meddeb, T. Nury, A. Badreddine, E.M. Karym, R. Sghaier, L. Bretillon, S. Guyot, M. Samadi, M. Cherkaoui-Malki, B. Nasser, M. Mejri, S. Ben-Hammou, M. Hammami, G. Lizard, Comparison of the effects of major fatty acids present in the Mediterranean diet (oleic acid, docosahexaenoic acid) and in hydrogenated oils (elaidic acid) on 7-ketocholesterol-induced oxiapoptophagy in microglial BV-2 cells, Chem. Phys. Lipids 207 (2017) 151-170.

[41] V. Mutemberezi, B. Buisseret, J. Masquelier, O. Guillemot-Legris, M. Alhouayek, G.G. Muccioli, Oxysterol levels and metabolism in the course of neuroinflammation: insights from in vitro and in vivo models, J. Neuroinflammation 15 (2018) 74 .

[42] V. Mutemberezi, J. Masquelier, O. Guillemot-Legris, G.G. Muccioli, Development and validation of an HPLC-MS method for the simultaneous quantification of key oxysterols, endocannabinoids, and ceramides: variations in metabolic syndrome, Anal. Bioanal. Chem. 408 (2016) 733-745.

[43] M. Ishikawa, T. Yoshitomi, D.F. Covey, C.F. Zorumski, Y. Izumi, Neurosteroids and oxysterols as potential therapeutic agents for glaucoma and Alzheimer's disease, Neuropsychiatry (London) 8 (2018) 344-359.

[44] K. Nieweg, H. Schaller, F.W. Pfrieger, Marked differences in cholesterol synthesis between neurons and glial cells from postnatal rats, J. Neurochem. 109 (2009) 125-134.

[45] M. Doria, L. Maugest, T. Moreau, G. Lizard, A. Vejux, Contribution of cholesterol and oxysterols to the pathophysiology of Parkinson's disease, Free Radic. Biol. Med. 101 (2016) 393-400. 
[46] A. Rutkowska, A.W. Sailer, K.K. Dev, EBI2 receptor regulates myelin development and inhibits LPC-induced demyelination, J. Neuroinflammation 14 (2017) 250.

[47] J. Makoukji, G. Shackleford, D. Meffre, J. Grenier, P. Liere, J.M. Lobaccaro, M. Schumacher, C. Massaad, Interplay between LXR and Wnt/beta-catenin signaling in the negative regulation of peripheral myelin genes by oxysterols, J. Neurosci. 31 (2011) 9620-9629.

[48] R. Loera-Valencia, J. Goikolea, C. Parrado-Fernandez, P. Merino-Serrais, S. Maioli, Alterations in cholesterol metabolism as a risk factor for developing Alzheimer's disease: potential novel targets for treatment, J. Steroid Biochem. Mol. Biol. 190 (2019) 104-114.

[49] A. Zarrouk, M. Hammami, T. Moreau, G. Lizard, Accumulation of 24S-hydroxycholesterol in neuronal SK-N-BE cells treated with hexacosanoic acid (C26:0): argument in favor of 24S-hydroxycholesterol as a potential biomarker of neurolipotoxicity, Rev Neurol (Paris) 171 (2015) 125-129.

[50] F. Gosselet, J. Saint-Pol, L. Fenart, Effects of oxysterols on the blood-brain barrier: implications for Alzheimer's disease, Biochem. Biophys. Res. Commun. 446 (2014) 687-691.

[51] I. Björkhem, M. Heverin, V. Leoni, S. Meaney, U. Diczfalusy, Oxysterols and Alzheimer's disease, Acta Neuronal Scand 114 (2006) 43-49.

[52] J. Saint-Pol, P. Candela, M.C. Boucau, L. Fenart, F. Gosselet, Oxysterols decrease apical-to-basolateral transport of Ass peptides via an ABCB1-mediated process in an in vitro Blood-brain barrier model constituted of bovine brain capillary endothelial cells, Brain Res. 1517 (2013) 1-15.

[53] F. Djelti, J. Braudeadu, E. Hudry, M. Dhenain, J. Varin, I. Bièche, C. Marquer, F. Chali, S. Ayciriex, N. Auzeil, S. Alves, D. Langui, M.C. Potier, O. Laprevote, M. Vidaud, C. Duyckaerts, R. Miles, P. Aubourg, N. Cartier, CYP46A1 inhibition, brain cholesterol accumulation and neurodegeneration pave the way for Alzheimer's disease, Brain 138 (2015) 2383-2398.

[54] W.J. Griffiths, J. Abdel-Khalik, E. Yutuc, G. Roman, M. Warner, J.A. Gustafsson, Y. Wang, Concentrations of bile acid precursors in cerebrospinal fluid of Alzheimer's disease patients, Free Radic. Biol. Med. 134 (2019) 42-52.

[55] G. Testa, D. Rossin, G. Poli, F. Biasi, G. Leonarduzzi, Implication of oxysterols in chronic inflammatory human diseases, Biochimie 153 (2018) 220-231.

[56] J.S. Park, R.L. Davis, C.M. Sue, Mitochondrial dysfunction in parkinson's disease: new mechanistic insights and therapeutic perspectives, Curr. Neurol. Neurosci. Rep. 18 (2018) 21.

[57] H. Jin, A. Kanthasamy, A. Ghosh, V. Anantharam, B. Kalyanaraman, A.G. Kanthasamy, Mitochondria-targeted antioxidants for treatment of Parkinson's disease: preclinical and clinical outcomes, Biochim. Biophys. Acta 1842 (2014) $1282-1294$.

[58] V. Cardenia, M.T. Rodriguez-Estrada, E. Boselli, G. Lercker, Cholesterol photosensitized oxidation in food and biological systems, Biochimie 95 (2013) 473-481.

[59] A. Zmyslowski, A. Szterk, Oxysterols as a biomarker in diseases, Clin. Chim. Acta 491 (2019) 103-113.

[60] J.P. Rantham Prabhakara, G. Feist, S. Thomasson, A. Thompson, E. Schommer, O. Ghribi, Differential effects of 24-hydroxycholesterol and 27-hydroxycholesterol on tyrosine hydroxylase and alpha-synuclein in human neuroblastoma SH-SY5Y cells, J. Neurochem. 107 (2008) 1722-1729.

[61] G. Marwarha, T. Rhen, T. Schommer, O. Ghribi, The oxysterol 27-hydroxycholesterol regulates alpha-synuclein and tyrosine hydroxylase expression levels in human neuroblastoma cells through modulation of liver $\mathrm{X}$ receptors and estrogen receptors--relevance to Parkinson's disease, J. Neurochem. 119 (2011) 1119-1136.

[62] I. Bjorkhem, A. Lovgren-Sandblom, V. Leoni, S. Meaney, L. Brodin, L. Salveson, K. Winge, S. Palhagen, P. Svenningsson, Oxysterols and Parkinson's disease: evidence that levels of 24S-hydroxycholesterol in cerebrospinal fluid correlates with the duration of the disease, Neurosci. Lett. 555 (2013) 102-105.

[63] S.E. Seidl, J.A. Santiago, H. Bilyk, J.A. Potashkin, The emerging role of nutrition in Parkinson's disease, Front. Aging Neurosci. 6 (2014) 36.

[64] V. Chiurchiu, A. Orlacchio, M. Maccarrone, Is modulation of oxidative stress an answer? The state of the art of redox therapeutic actions in neurodegenerative diseases, Oxid. Med. Cell. Longev. 2016 (2016), 7909380.

[65] L. Houdebine, C.A. Gallelli, M. Rastelli, N.K. Sampathkumar, J. Grenier, Effect of physical exercise on brain and lipid metabolism in mouse models of multiple sclerosis, Chem. Phys. Lipids 207 (2017) 127-134.

[66] S. Zhornitsky, K.A. McKay, L.M. Metz, C.E. Teunissen, M. Rangachari, Cholesterol and markers of cholesterol turnover in multiple sclerosis: relationship with disease outcomes, Mult. Scler. Relat. Disord. 5 (2016) 53-65.

[67] F. Chalmin, V. Rochemont, C. Lippens, A. Clottu, A.W. Sailer, D. Merkler, S. Hugues, C. Pot, Oxysterols regulate encephalitogenic CD4(+) T cell trafficking during central nervous system autoimmunity, J. Autoimmun. 56 (2015) 45-55.

[68] C. van de Kraats, J. Killestein, V. Popescu, E. Rijkers, H. Vrenken, D. Lutjohann, F. Barkhof, C.H. Polman, C.E. Teunissen, Oxysterols and cholesterol precursors correlate to magnetic resonance imaging measures of neurodegeneration in multiple sclerosis, Mult. Scler. 20 (2014) 412-417.

[69] S. Vuletic, H. Kennedy, J.J. Albers, J. Killestein, H. Vrenken, D. Lutjohann, C.E. Teunissen, Cerebrospinal fluid apolipoprotein E and phospholipid transfer protein activity are reduced in multiple sclerosis; relationships with the brain MRI and CSF lipid variables, Mult. Scler. Relat. Disord. 3 (2014) 533-541.

[70] C.E. Teunissen, C.D. Dijkstra, C.H. Polman, E.L. Hoogervorst, K. von Bergmann, D. Lutjohann, Decreased levels of the brain specific $24 \mathrm{~S}$-hydroxycholesterol and cholesterol precursors in serum of multiple sclerosis patients, Neurosci. Lett. 347 (2003) 159-162.
[71] A. Vejux, A. Namsi, T. Nury, T. Moreau, G. Lizard, Biomarkers of amyotrophic lateral sclerosis: current status and interest of oxysterols and phytosterols, Front. Mol. Neurosci. 11 (2018) 12.

[72] M. Bezine, A. Namsi, R. Sghaier, R. Ben Khalifa, H. Hamdouni, F. Brahmi, I. Badreddine, W. Mihoubi, T. Nury, A. Vejux, A. Zarrouk, J. de Seze, T. Moreau, B. Nasser, G. Lizard, The effect of oxysterols on nerve impulses, Biochimie 153 (2018) 46-51.

[73] A.M. Malfitano, G. Marasco, M.C. Proto, C. Laezza, P. Gazzerro, M. Bifulco, Statins in neurological disorders: an overview and update, Pharmacol. Res. 88 (2014) 74-83.

[74] A.M. Giudetti, A. Romano, A.M. Lavecchia, S. Gaetani, The role of brain cholesterol and its oxidized products in alzheimer's disease, Curr. Alzheimer Res. 13 (2016) 198-205.

[75] V. Cardenia, M.T. Rodriguez-Estrada, A. Lorenzini, E. Bandini, C. Angeloni, S. Hrelia, M. Malaguti, Effect of broccoli extract enriched diet on liver cholesterol oxidation in rats subjected to exhaustive exercise, J. Steroid Biochem. Mol. Biol. 169 (2017) 137-144.

[76] G.P. Bates, R. Dorsey, J.F. Gusella, M.R. Hayden, C. Kay, B.R. Leavitt, M. Nance, C.A. Ross, R.I. Scahill, R. Wetzel, E.J. Wild, S.J. Tabrizi, Huntington disease, Nat. Rev. Dis. Primers 1 (2015) 15005.

[77] F.O. Walker, Huntington's disease, Lancet 369 (2007) 218-228.

[78] M. Shankaran, E. Di Paolo, V. Leoni, C. Caccia, C. Ferrari Bardile, H. Mohammed, S. Di Donato, S. Kwak, D. Marchionini, S. Turner, E. Cattaneo, M. Valenza, Early and brain region-specific decrease of de novo cholesterol biosynthesis in Huntington's disease: a cross-validation study in Q175 knock-in mice, Neurobiol. Dis. 98 (2017) 66-76.

[79] M. Valenza, J.B. Carroll, V. Leoni, L.N. Bertram, I. Bjorkhem, R.R. Singaraja, S. Di Donato, D. Lutjohann, M.R. Hayden, E. Cattaneo, Cholesterol biosynthesis pathway is disturbed in YAC128 mice and is modulated by huntingtin mutation, Hum. Mol. Genet. 16 (2007) 2187-2198.

[80] M. Valenza, V. Leoni, A. Tarditi, C. Mariotti, I. Bjorkhem, S. Di Donato, E. Cattaneo, Progressive dysfunction of the cholesterol biosynthesis pathway in the R6/2 mouse model of Huntington's disease, Neurobiol. Dis. 28 (2007) 133-142.

[81] C.M. Chen, Y.R. Wu, M.L. Cheng, J.L. Liu, Y.M. Lee, P.W. Lee, B.W. Soong, D.T. Chiu, Increased oxidative damage and mitochondrial abnormalities in the peripheral blood of Huntington's disease patients, Biochem. Biophys. Res. Commun. 359 (2007) 335-340.

[82] W.J. Griffiths, Y. Wang, Oxysterol research: a brief review, Biochem. Soc. Trans. 47 (2019) 517-526.

[83] M.M. Essa, M. Moghadas, T. Ba-Omar, M. Walid Qoronfleh, G.J. Guillemin, T. Manivasagam, A. Justin-Thenmozhi, B. Ray, A. Bhat, S.B. Chidambaram, A.J. Fernandes, B.J. Song, M. Akbar, Protective effects of antioxidants in Huntington's disease: an extensive review, Neurotox. Res. 35 (2019) 739-774.

[84] J. Gil-Mohapel, P.S. Brocardo, B.R. Christie, The role of oxidative stress in Huntington's disease: are antioxidants good therapeutic candidates?, Curr. Drug Targets 15 (2014) 454-468.

[85] I. Tunez, F. Sanchez-Lopez, E. Aguera, R. Fernandez-Bolanos, F.M. Sanchez, I. Tasset-Cuevas, Important role of oxidative stress biomarkers in Huntington's disease, J. Med. Chem. 54 (2011) 5602-5606.

[86] A. Goswami, P. Dikshit, A. Mishra, S. Mulherkar, N. Nukina, N.R. Jana, Oxidative stress promotes mutant huntingtin aggregation and mutant huntingtin-dependent cell death by mimicking proteasomal malfunction, Biochem. Biophys. Res. Commun. 342 (2006) 184-190.

[87] E.C. Stack, W.R. Matson, R.J. Ferrante, Evidence of oxidant damage in Huntington's disease: translational strategies using antioxidants, Ann. N. Y. Acad. Sci. 1147 (2008) 79-92

[88] H.L. Paulson, N.M. Bonini, K.A. Roth, Polyglutamine disease and neuronal cell death, Proc Natl. Acad. Sci. U. S. A. 97 (2000) 12957-12958.

[89] I. Tunez, I. Tasset, V. Perez-De La Cruz, A. Santamaria, 3-Nitropropionic acid as a tool to study the mechanisms involved in Huntington's disease: past, present and future, Molecules 15 (2010) 878-916.

[90] C.D. Keene, C.M. Rodrigues, T. Eich, C. Linehan-Stieers, A. Abt, B.T. Kren, C.J. Steer, W.C. Low, A bile acid protects against motor and cognitive deficits and reduces striatal degeneration in the 3-nitropropionic acid model of Huntington's disease, Exp. Neurol. 171 (2001) 351-360.

[91] P. Kumar, A. Kumar, Effect of lycopene and epigallocatechin-3-gallate against 3-nitropropionic acid induced cognitive dysfunction and glutathione depletion in rat: a novel nitric oxide mechanism, Food Chem. Toxicol. 47 (2009) 2522-2530.

[92] B.N. Al-Sabahi, M.O. Fatope, M.M. Essa, S. Subash, S.N. Al-Busafi, F.S. Al-Kusaibi, T. Manivasagam, Pomegranate seed oil: effect on 3-nitropropionic acid-induced neurotoxicity in PC12 cells and elucidation of unsaturated fatty acids composition, Nutr. Neurosci. 20 (2017) 40-48.

[93] T. Velusamy, A.S. Panneerselvam, M. Purushottam, M. Anusuyadevi, P.K. Pal, S. Jain, M.M. Essa, G.J. Guillemin, M. Kandasamy, Protective effect of antioxidants on neuronal dysfunction and plasticity in Huntington's disease, Oxid. Med. Cell. Longev. 2017 (2017), 3279061.

[94] T. Nury, A. Zarrouk, K. Ragot, M. Debbabi, J.M. Riedinger, A. Vejux, P. Aubourg, G. Lizard, 7-Ketocholesterol is increased in the plasma of X-ALD patients and induces peroxisomal modifications in microglial cells: potential roles of 7-ketocholesterol in the pathophysiology of X-ALD, J. Steroid Biochem. Mol. Biol. 169 (2017) 123-136.

[95] S. Petrillo, F. Piemonte, A. Pastore, G. Tozzi, C. Aiello, A. Pujol, M. Cappa, E. Bertini, Glutathione imbalance in patients with X-linked adrenoleukodystrophy, Mol. Genet. Metab. 109 (2013) 366-370. 
[96] M. Baarine, K. Ragot, A. Athias, T. Nury, Z. Kattan, E.C. Genin, P. Andreoletti, F. Menetrier, J.M. Riedinger, M. Bardou, G. Lizard, Incidence of Abcd1 level on the induction of cell death and organelle dysfunctions triggered by very long chain fatty acids and TNF-alpha on oligodendrocytes and astrocytes, Neurotoxicology 33 (2012) 212-228.

[97] P. Palozza, R. Simone, A. Catalano, G. Monego, A. Barini, M.C. Mele, N. Parrone S. Trombino, N. Picci, F.O. Ranelletti, Lycopene prevention of oxysterol-induced proinflammatory cytokine cascade in human macrophages: inhibition of NF-kap$\mathrm{paB}$ nuclear binding and increase in PPARgamma expression, J. Nutr. Biochem. 22 (2011) 259-268.

[98] J. Jang, S. Park, H. Jin Hur, H.J. Cho, I. Hwang, Y. Pyo Kang, I. Im, H. Lee, E. Lee, W. Yang, H.C. Kang, S. Won Kwon, J.W. Yu, D.W. Kim, 25-hydroxycholesterol contributes to cerebral inflammation of X-linked adrenoleukodystrophy through activation of the NLRP3 inflammasome, Nat. Commun. 7 (2016) 13129.

[99] S. Fourcade, M. Ruiz, C. Guilera, E. Hahnen, L. Brichta, A. Naudi, M. Portero-Otin, G. Dacremont, N. Cartier, R. Wanders, S. Kemp, J.L. Mandel, B. Wirth, R. Pamplona, P. Aubourg, A. Pujol, Valproic acid induces antioxidant effects in X-linked adrenoleukodystrophy, Hum. Mol. Genet. 19 (2010) 2005-2014.

[100] J. Lopez-Erauskin, S. Fourcade, J. Galino, M. Ruiz, A. Schluter, A. Naudi, M. Jove, M. Portero-Otin, R. Pamplona, I. Ferrer, A. Pujol, Antioxidants halt axonal degeneration in a mouse model of X-adrenoleukodystrophy, Ann. Neurol. 70 (2011) 84-92.

[101] A. Zarrouk, T. Nury, M. Samadi, Y. O'Callaghan, M. Hammami, N.M. O'Brien, G. Lizard, J.J. Mackrill, Effects of cholesterol oxides on cell death induction and calcium increase in human neuronal cells (SK-N-BE) and evaluation of the protective effects of docosahexaenoic acid (DHA; C22:6 n-3), Steroids 99 (2015) 238-247.

[102] M.C. Royer, S. Lemaire-Ewing C. Desrumaux, S. Monier, J.P. Pais de Barros, A. Athias, D. Neel, L. Lagrost, 7-ketocholesterol incorporation into sphingolipid/cholesterol-enriched (lipid raft) domains is impaired by vitamin E: a specific role for alpha-tocopherol with consequences on cell death, J. Biol. Chem. 284 (2009) 15826-15834.

[103] T. Nury, R. Sghaier, A. Zarrouk, F. Menetrier, T. Uzun, V. Leoni, C. Caccia, W. Meddeb, A. Namsi, K. Sassi, W. Mihoubi, J.M. Riedinger, M. Cherkaoui-Malki, T. Moreau, A. Vejux, G. Lizard, Induction of peroxisomal changes in oligodendrocytes treated with 7-ketocholesterol: attenuation by alpha-tocopherol, Biochimie 153 (2018) 181-202.

[104] G. Testa, P. Gamba, U. Badilli, S. Gargiulo, M. Maina, T. Guina, S. Calfapietra, F. Biasi, R. Cavalli, G. Poli, G. Leonarduzzi, Loading into nanoparticles improves quercetin's efficacy in preventing neuroinflammation induced by oxysterols, PLoS One 9 (2014), e96795.

[105] A. Kim, Y.J. Nam, C.S. Lee, Taxifolin reduces the cholesterol oxidation product-induced neuronal apoptosis by suppressing the Akt and NF-kappaB activation-mediated cell death, Brain Res. Bull. 134 (2017) 63-71.

[106] D.H. Lee, Y.J. Nam, C.S. Lee, Quercetin-3-O-(2"-galloyl)-alpha-L-rhamnopyranoside attenuates cholesterol oxidation product-induced apoptosis by suppressing NF-kappaB-mediated cell death process in differentiated PC12 cells, Naunyn Schmiedebergs Arch. Pharmacol. 388 (2015) 869-881.

[107] J. Vina, F. Sanchis-Gomar, V. Martinez-Bello, M.C. Gomez-Cabrera, Exercise acts as a drug; the pharmacological benefits of exercise, Br. J. Pharmacol. 167 (2012) $1-12$
[108] M.W. Voss, C. Vivar, A.F. Kramer, H. van Praag, Bridging animal and human models of exercise-induced brain plasticity, Trends Cogn. Sci. (Regul. Ed.) 17 (2013) 525-544.

[109] S. Colcombe, A.F. Kramer, Fitness effects on the cognitive function of older adults: a meta-analytic study, Psychol. Sci. 14 (2003) 125-130.

[110] F. Gomez-Pinilla, L. Dao, V. So, Physical exercise induces FGF-2 and its mRNA in the hippocampus, Brain Res. 764 (1997) 1-8.

[111] Z. Radak, A. Toldy, Z. Szabo, S. Siamilis, C. Nyakas, G. Silye, J. Jakus, S. Goto, The effects of training and detraining on memory, neurotrophins and oxidative stress markers in rat brain, Neurochem. Int. 49 (2006) 387-392.

[112] E.J. Lesnefsky, Q. Chen, B. Tandler, C.L. Hoppel, Mitochondrial dysfunction and myocardial ischemia-reperfusion: implications for novel therapies, Annu. Rev. Pharmacol. Toxicol. 57 (2017) 535-565.

[113] K.A. Webster, Mitochondrial membrane permeabilization and cell death during myocardial infarction: roles of calcium and reactive oxygen species, Future Cardiol. 8 (2012) 863-884

[114] S. Paradis, V. Leoni, C. Caccia, A. Berdeaux, D. Morin, Cardioprotection by the TSPO ligand 4'-chlorodiazepam is associated with inhibition of mitochondrial accumulation of cholesterol at reperfusion, Cardiovasc. Res. 98 (2013) 420-427.

[115] D. Morin, J. Musman, S. Pons, A. Berdeaux, B. Ghaleh, Mitochondrial translocator protein (TSPO): from physiology to cardioprotection, Biochem. Pharmacol. 105 (2016) 1-13.

[116] J. Musman, S. Pons, C. Barau, C. Caccia, V. Leoni, A. Berdeaux, B. Ghaleh, D. Morin, Regular treadmill exercise inhibits mitochondrial accumulation of cholesterol and oxysterols during myocardial ischemia-reperfusion in wild-type and ob/ ob mice, Free Radic. Biol. Med. 101 (2016) 317-324.

[117] G.S. Ferreira, P.R. Pinto, R.T. Iborra, V. Del Bianco, M.F.M. Santana, E.R. Nakandakare, V.S. Nunes, C.E. Negrao, S. Catanozi, M. Passarelli, Aerobic exercise training selectively changes oxysterol levels and metabolism reducing cholesterol accumulation in the aorta of dyslipidemic mice, Front. Physiol. 8 (2017) 644

[118] P.R. Pinto, D.D. Rocco, L.S. Okuda, A. Machado-Lima, G. Castilho, K.S. da Silva, D.J. Gomes, S. Pinto Rde, R.T. Iborra, S. Ferreira Gda, E.R. Nakandakare, U.F. Machado, M.L. Correa-Giannella, S. Catanozi, M. Passarelli, Aerobic exercise training enhances the in vivo cholesterol trafficking from macrophages to the liver independently of changes in the expression of genes involved in lipid flux in macrophages and aorta, Lipids Health Dis. 14 (2015) 109.

[119] A.R. Cho, J.Y. Moon, S. Kim, K.Y. An, M. Oh, J.Y. Jeon, D.H. Jung, M.H. Choi, J.W. Lee, Effects of alternate day fasting and exercise on cholesterol metabolism in overweight or obese adults: a pilot randomized controlled trial, Metabolism 93 (2019) 52-60.

[120] S. Ayers, J. Baer, M.T. Ravi Subbiah, Exercised-induced increase in lipid peroxidation parameters in amenorrheic female athletes, Fertil. Steril. 69 (1998) 73-77.

[121] N. Feter, M.P. Freitas, N.G. Gonzales, D. Umpierre, R.K. Cardoso, A.J. Rombaldi, Effects of physical exercise on myelin sheath regeneration: a systematic review and meta-analysis, Sci. Sports 33 (2018) 8-21.

[122] S.A. Durazo, U.B. Kompella, Functionalized nanosystems for targeted mitochondrial delivery, Mitochondrion 12 (2012) 190-201.

[123] E. Berzabá-Evoli, J.G. Tejas-Juárez, N.P. Gómez-Crisóstomo, E.N. De la Cruz-Hernández, E. Martínez-Abundis, Chemicals with mitochondrial targets for the treatment of neurodegenerative disorders, Annu. Res. Rev. Biol. 21 (2017) 1-19. 